\title{
Inhibitory Mechanism by Polysialic Acid for Lamina-Specific Branch Formation of Thalamocortical Axons
}

\author{
Nobuhiko Yamamoto,, ${ }^{1}$ Kayo Inui,, ${ }^{1}$ Yuki Matsuyama, ${ }^{1}$ Akiko Harada, ${ }^{1}$ Kenji Hanamura, ${ }^{1}$ Fujio Murakami, ${ }^{1}$ \\ Edward S. Ruthazer, ${ }^{1}$ Urs Rutishauser, ${ }^{2}$ and Tatsunori Seki ${ }^{3}$ \\ ${ }_{1}^{1}$ Division of Biophysical Engineering, Graduate School of Engineering Science, Osaka University, Toyonaka, Osaka 560- \\ 8531, Japan, 2Program in Cellular Biochemistry and Biophysics, Memorial Sloan-Kettering Cancer Center, New York, \\ New York 10021, and '3epartment of Anatomy, Juntendo University, School of Medicine, Hongo, Bunkyo-ku, Tokyo \\ 113-8421, Japan
}

\begin{abstract}
During development, thalamocortical axons form arbors primarily in layer 4 of the neocortex. This lamina-specific branch formation was studied in cultures of rat thalamic explants grown next to chemically fixed cortical slices. After a week in vitro, thalamic axons formed branches specifically in the target layer of fixed cortical slices, regardless of the orientation of the ingrowth. This in vitro system permits a direct assessment of contributions of membrane-associated molecules to thalamic axon branch formation. To this end, the present study uses three enzymatic perturbations: chondroitinase, phosphatidylinositol phospholipase $\mathrm{C}$, or the polysialic acid (PSA)-specific endoneuraminidase (endo $\mathrm{N}$ ). With endo $\mathrm{N}$ pretreatment of cortex, the number of branch points was increased significantly, whereas branch tip
\end{abstract}

length was decreased. In addition, the localization of branch points to the target layer was weakened considerably. These features of branch formation were not altered by the other two enzymatic treatments, except that branch tips were shortened by chondroitinase treatment to the same extent as in endo $\mathrm{N}$ treatment. These results suggest that membrane-bound components are involved in lamina-specific branch formation of thalamocortical axons, and in particular that PSA moieties contribute to laminar specificity by inhibiting branch emergence in inappropriate layers.

Key words: thalamocortical projection; axonal branch; layer specificity; cortical development; target recognition; axon guidance; thalamus
One of the key issues in neurobiology is how axons form elaborate branches within their target tissues. The thalamocortical projection in the mammalian brain is a suitable system to address this issue because its layer-specific projection pattern is well characterized (Jones, 1981; Gilbert, 1983). In development, sensory thalamic axons, after reaching the cortex, extend perpendicularly to the cortical layers and form terminal branches predominantly in layer 4 (Lund and Mustari, 1977; Ghosh and Shatz, 1992; Agmon et al., 1993; Kageyama and Robertson, 1993; Catalano et al., 1996). Previous studies using organotypic culture methods have demonstrated that even in vitro, thalamic axons form branches selectively in layer 4 (Yamamoto et al., 1989, 1992; Molnár and Blakemore, 1991; Bolz et al., 1992). Time lapse imaging of thalamocortical axons in this system has further revealed that the initial branches appear in the target layer and that branching and axonal stop behaviors do not always occur together (Yamamoto et al., 1997). These findings raise the possibility that branch formation is regulated by local cues, possibly membrane-associated molecules, which are independent of those governing axonal termination (Yamamoto et al., 2000).

Recent studies have suggested that particular guidance molecules can regulate branch formation. In cortical circuits, ephrin-A5, a ligand of Eph receptors, is expressed in a lamina-specific manner (Gao et al., 1998; Donoghue and Rakic, 1999; Mackarehtschian et al., 1999) and has been shown to induce branch buds from a subset of cortical axons (Castellani et al., 1998). A slit protein, which was

\footnotetext{
Received March 24, 2000; revised Aug. 2, 2000; accepted Sept. 15, 2000.

This work was supported by Grants-in-Aid for Scientific Research Projects 08254207 and 09680793 from Japanese Ministry of Education, Culture, and Sports, and from Core Research for Evolutional Science and Technology. We thank Dr. R. Shirasaki for helpful comments on this manuscript.

Correspondence should be addressed to Nobuhiko Yamamoto, Division of Biophysical Engineering, Graduate School of Engineering Science, Osaka University, Toyonaka, Osaka 560-8531, Japan. E-mail: nob-yama@bpe.es.osaka-u.ac.jp.

Dr. Ruthazer's present address: 1 Bungtown Road/Beckman, Cold Spring Harbor Laboratory, Cold Spring Harbor, NY 11724.

Copyright (C) 2000 Society for Neuroscience $0270-6474 / 00 / 209145-07 \$ 15.00 / 0$
}

originally identified as a repellent molecule for axons, has also been shown to increase the number of axonal branches from spinal ganglion neurons (Wang et al., 1999). However, the extent to which these molecules are responsible for branching per se is not known because these proteins also regulate axonal growth. On the other hand, cellular interactions mediated by adhesion molecules are also likely to contribute to axonal branching. It has been demonstrated that polysialic acid (PSA), the sugar moiety attached to the neural cell adhesion molecule (NCAM), can regulate axon fasciculation in motor pathways (Tang et al., 1994). Regulation of branching in axon tracts has also been observed in the CNS (Yin et al., 1995; Daston et al., 1996; Seki and Rutishauser, 1998). Nevertheless, it is not known how PSA controls terminal branching of individual axons.

In this study we investigated the characteristics of branchregulating molecules using cultures of thalamic explants juxtaposed to chemically fixed cortical slices. Thus, membrane-bound components in the developing cortex were retained in the absence of diffusible factors released from cortical cells. Furthermore, the biochemical properties of presumptive factors could be analyzed by applying enzymes that specifically degrade individual classes of surface molecules in the fixed cortical slices. The result suggests that an inhibitory mechanism acting through PSA expressed by the cortex plays a role in layer-specific branch formation.

Part of this study has been reported in a preliminary form (Yamamoto et al., 1998).

\section{MATERIALS AND METHODS}

Preparation of fixed cortical slices and enzymatic treatments. The methods of fixation of cortical slices and enzymatic treatments have been described elsewhere (Yamamoto et al., 2000). In brief, the occipital cortex was dissected from P7 rat pups (Sprague Dawley) and then cut into $250-\mu \mathrm{m}-$ thick slices. These slices were immersed in a solution containing 3.5\% paraformaldehyde for $3 \mathrm{hr}$ at $4^{\circ} \mathrm{C}$. After extensive washes, these slices were incubated in an enzyme solution of either chondroitinase $A B C(5 \mathrm{U} / \mathrm{ml})$ or PSA-specific endoneuraminidase (endo N) (1:2000 in HBSS) for 8-9 hr, followed by several washes before culturing with living thalamic explants. Cortical slices were fixed before enzymatic treatment because this incuba- 
tion duration, although necessary for adequate enzymatic removal of the various substrates (see below), is too long for living slices to endure. Phosphatidylinositol phospholipase C (PI-PLC; $0.5-1 \mathrm{U} / \mathrm{ml})$ was applied for $15 \mathrm{~min}$ to living cortical slices, because this enzyme failed to remove glycosyl-phophatidylinositol (GPI)-anchored molecules after fixation. PIPLC-treated slices were fixed and washed as above after enzymatic treatment. The efficacy of these enzyme treatments was confirmed by an immunohistochemical method. Untreated and treated cortical slices were cut into thin sections $(16-20 \mu \mathrm{m})$ and incubated with monoclonal antibodies against chondroitin sulfate (CS56; 1:50-100; Seikagakukogyo, Tokyo, Japan), PSA (12E3, 1:1000; Seki and Arai, 1991), or TAG-1 (4D7; a gift from Dr. M. Yamamoto, University of Tsukuba) and followed by incubation with Cy3-conjugated secondary antibodies for visualization.

Culture of living thalamic explant with the fixed cortical slice. The dorsal thalamic region containing the lateral geniculate nucleus was dissected from E15 rat embryos and was cultured with fixed cortical slices or with enzyme-treated fixed slices on collagen-coated membranes in serum-free, hormone-supplemented medium (Yamamoto et al., 1989, 1992). The thalamic explant was placed at either the ventricular side or pial side of the fixed cortical slice. In the ventricular arrangement, the white matter or layer 6 was often removed from the fixed cortical slices to compensate for the fact that only a small number of axons reached layer 4 in the fixed slices (Yamamoto et al., 2000). The cultures were maintained at $37^{\circ} \mathrm{C}$ in an environment of humidified $95 \%$ air and $5 \% \mathrm{CO}_{2}$.

Axonal labeling and quantitative analysis of branching. After 1 week in vitro, the cultures were fixed with $4 \%$ paraformaldehyde in $0.1 \mathrm{M}$ phosphate buffer. To label thalamic axons, a small crystal of 1,1'-dioctadecyl-3,3,3',3' tetramethyl indocarbocyanine perchlorate (DiI; Molecular Probes, Eugene, OR) was inserted at a few locations in the thalamic explant (Godement et al., 1987).

Several days after DiI implantation, these samples were observed by confocal microscopy. The $20 \times$ objective lens and a filter set for rhodamine were used to visualize DiI-labeled axons. A series of images was collected at different depths $(2.5-5 \mu \mathrm{m}$ interval) and superimposed. Each image was averaged 10 times to increase the signal-to-noise ratio. Transparent images were also collected with a $4 \times$ objective lens to locate the pial surface of the cortical slices. The collected images obtained by confocal microscopy were stored on magneto-optical disks for the following analysis.

Individually distinguishable axons that reached or passed through layer 4 were selected and drawn using NIH Image software. Densely intermingled axons were ignored because it was impossible to trace them accurately. Then, the distances from the pial surface to every branch point and lengths of branches were measured in NIH Image using specialized macros.

After confocal microscopy, the slices were stained in a bisbenzamide solution (0.001-0.002\% in PBS) to observe laminar organization under an epifluorescence microscope. The top and bottom halves of the cell dense cortical plate were defined as layers $2 / 3$ and 4 , respectively.

Statistical analysis. Monte Carlo analysis was performed to test laminar localization of branch points. In this simulation, branches were generated at random along the length of a model axon extending perpendicular to the cortical layers. The axonal lengths and branch numbers were matched to those of the experimentally obtained axons. The probability of branching as a function of distance from the pial surface was calculated in $0.1 \mathrm{~mm}$ bins for 10,000 simulated arbors and compared with the observed probability in each bin.

\section{RESULTS}

\section{Branch formation of thalamic axons on fixed cortical slices}

The laminar location where thalamocortical axons formed branches was examined on P7 fixed cortical slices. At this stage, cortical lamination is almost complete. In addition, branch formation is abundant, although it is first observed at P3-P4 (Kageyama and Robertson, 1993).

First, the thalamic explant was placed at the ventricular side of the fixed cortical slice. In this ventricular arrangement, the orientation of axonal ingrowth is the same as that in vivo. After 1 week in vitro, most thalamic axons labeled with DiI exhibited branch formation in the fixed cortex comparable with that seen in living slices. Figure 1 shows an example of a thalamic axon forming branches. A striking feature was that the arbor was quite restricted to layer 4, whereas the axonal stem in the deep layers was straight from the ventricular surface (Fig. $1 A, B$ ). Such localization of branch formation was also observed in fixed slices in which layer 6 and the white matter had been removed. These cut slices were used in most cases, because thalamic axons entering from the ventricular surface barely reached the middle layer under the conditions of fixation used in this study. A total of 51 individually distinguishable axons were collected from 20 cultures ( 6 whole slices and 14 cut slices). A fraction of them (6 of 51) had no branches, but the
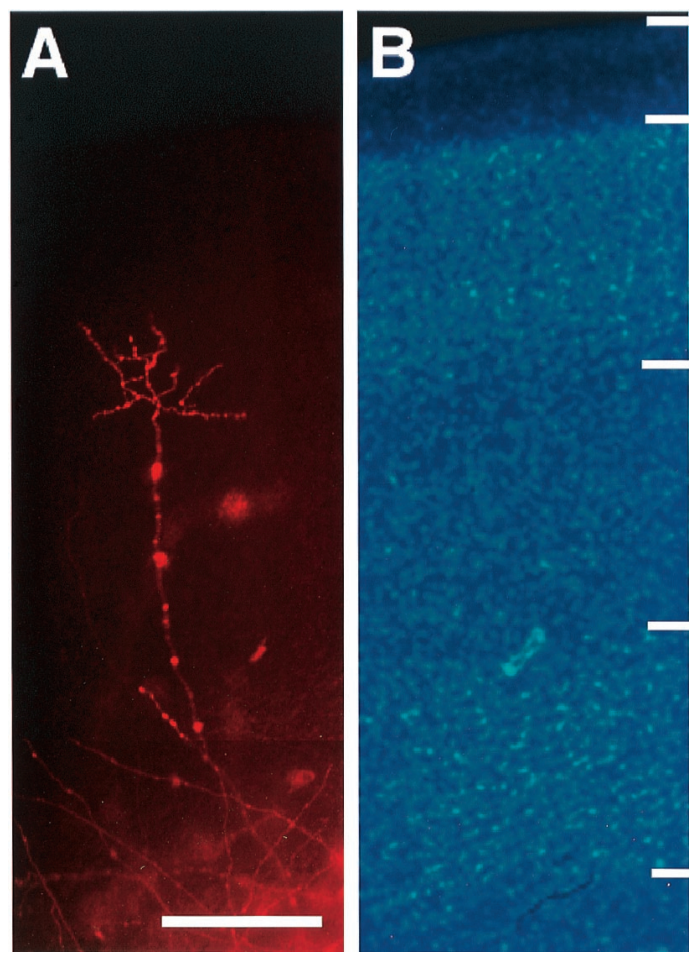

Figure 1. Branch formation of thalamic axons in a fixed cortical slice. $A$, Living thalamic explant was cultured at the ventricular side of a fixed P7 cortical slice. After $7 \mathrm{~d}$ in vitro cultures were fixed, and thalamic axons were labeled with DiI. $B$, Cortical lamination is shown by bisbenzamide staining. In $B$ the pial surface and the laminar boundaries between layers $1,2 / 3-4,5$, 6 , and the white matter are shown to the right side (white bars). The top and bottom halves of the cell dense layer were defined as layers $2 / 3$ and 4 , respectively. Scale bar: $A, 200 \mu \mathrm{m}$.

remaining axons formed branches in and around layer 4. The number of branches and the complexity were varied among samples (Fig. $2 A$ ). The number of branch points and the extent of arbor size were $3.7 \pm 0.5$ and $144 \pm 11 \mu \mathrm{m}$, respectively (Table 1 ).

The laminar locations of these branch points were examined quantitatively. As shown in Figure $2 B$, the majority of branch points were distributed in the range of $0.3-0.5 \mathrm{~mm}$ from the pial surface (Fig. 2B, hatched columns), which approximately corresponds to the average position of layer 4 as determined by the bisbenzamide staining of the 20 cultures. It seemed possible that the peak in the distribution may have been attributable to a sampling bias, because only axons that passed through or reached layer 4 were collected. To test this possibility, a Monte Carlo analysis was performed in which branches were generated randomly from experimentally obtained parent axons (see Materials and Methods). This analysis clearly showed that the observed distribution of branch points was significantly higher at $0.3-0.5 \mathrm{~mm}$ from the pial surface $(p<0.0001$ in the range of $0.3-0.4 \mathrm{~mm}$ and $p<0.001$ in $0.4-0.5 \mathrm{~mm}$ ) than that resulting from the simulation (Gaussian-like curves in Fig. 2B). This indicates that branching occurs preferentially at the narrow range of depths corresponding to layer 4. Moreover, this simulation showed that the frequency of branching in layer $5(0.6-0.7 \mathrm{~mm}$ from the pial surface) is lower than the value predicted by the simulation.

Previous studies using cocultures of thalamus with living cortex have indicated that such axonal branching is not dependent on the distance thalamic axons may have grown to reach the cortex or on the orientation of the ingrowth (Bolz et al., 1992; Yamamoto et al., 1997). To further confirm this for the present conditions, the thalamic explant was placed at the pial surface of the fixed cortical slice, and branching locations were examined in the same way. The observed axons also formed branches with laminar specificity, although branching took place in slightly more superficial layers (Fig. $3 A$ ). The distribution histogram of branch points (in 44 axons 


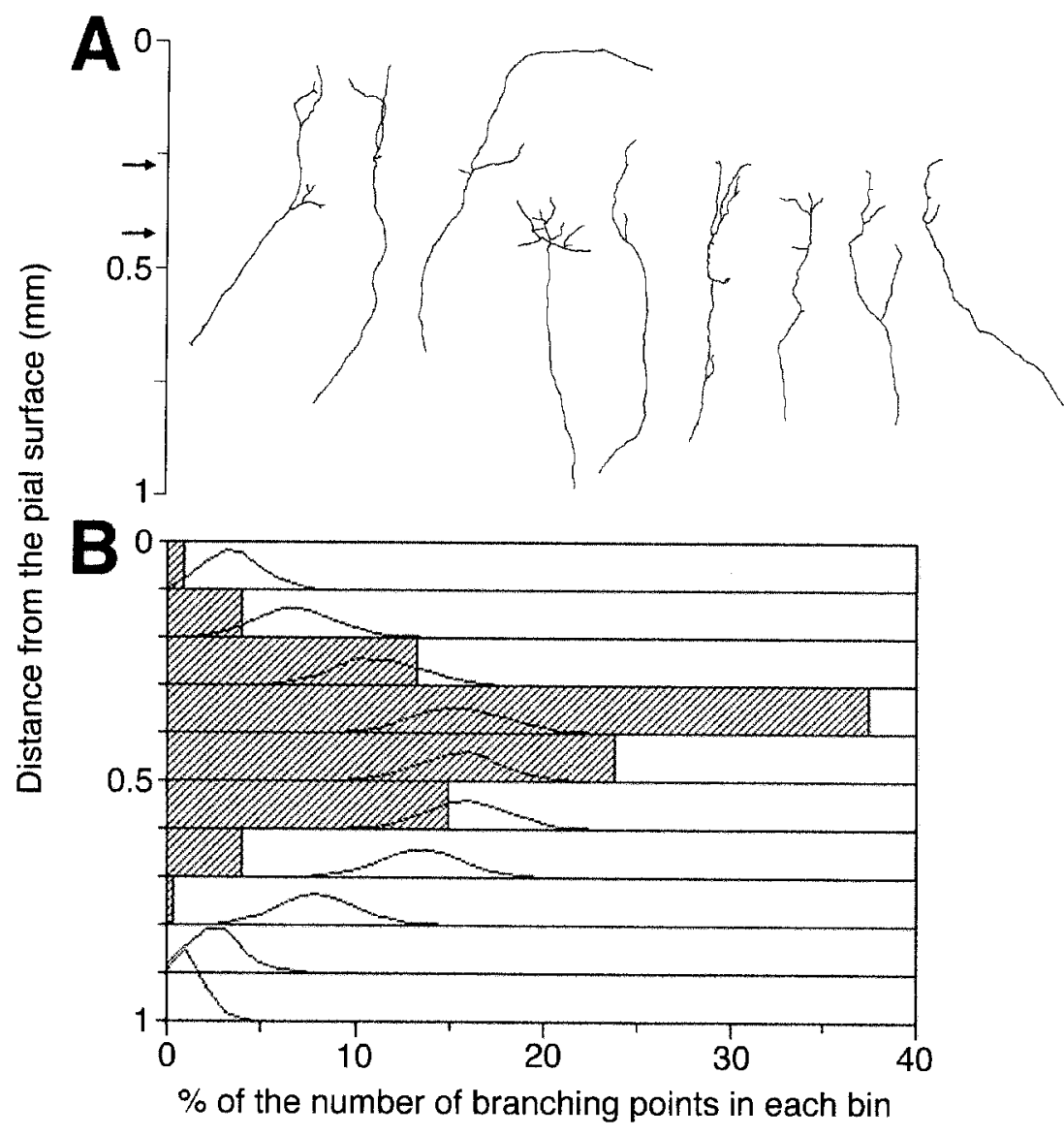

Figure 2. Laminar distribution of branch formation for ventricular ingrowth. $A$, Individually distinguishable DiIlabeled axons were traced from confocal images. Nine representative examples are shown from a total of 51 axons. Arrows indicate the average borders of layer 4. The borders were determined by bisbenzamide staining of fixed cortical slices $(n=20)$. B . Distribution histogram of branch points constructed with $0.1 \mathrm{~mm}$ bin (hatched columns). Gaussian-like curves represent the distribution of the probability that branches appear randomly from the shaft of experimentally obtained axons (51 axons, 10,000 trials). Note that experimentally obtained values $0.3-0.5$ $\mathrm{mm}$ from the pia are significantly larger $(p<0.0001$ in $0.3-0.4 \mathrm{~mm}$ from the pia and $p<0.001$ in $0.4-0.5 \mathrm{~mm}$ ) than those in the simulation.

Table 1. The number of branches and branch length in treated slices

\begin{tabular}{|c|c|c|c|c|}
\hline & Untreated & Ch'ase & PI-PLC & endo $\mathrm{N}$ \\
\hline Average \# of branches & $3.7 \pm 0.5(n=51)$ & $4.5 \pm 0.8(n=21)$ & $4.3 \pm 1.0(n=26)$ & $6.5 \pm 1.2^{a}(n=22)$ \\
\hline Branch tip length $(\mu \mathrm{m})$ & $89.5 \pm 7.7(n=233)$ & $48.5 \pm 5.3^{b}(n=113)$ & $61.4 \pm 7.2^{b}(n=146)$ & $45.4 \pm 4.7^{b}(n=163)$ \\
\hline Arbor size $(\mu \mathrm{m})$ & $144 \pm 11$ & $147 \pm 30$ & $156 \pm 22$ & $142 \pm 19$ \\
\hline Total length $(\mu \mathrm{m})$ & $1591 \pm 59.5$ & $1541 \pm 83.1$ & $1602 \pm 88.5$ & $1864 \pm 143$ \\
\hline
\end{tabular}

${ }^{a}$ Significant difference ( $p<0.05$, Mann-Whitney $U$ test).

${ }^{b}$ Significant difference $(p<0.001$, Student $t$ test). Arbor size is expressed as the horizontal extent of axonal branches. Total length is expressed as the total axon length including branches per unit length of the cortical plate perpendicular to the laminar structure. Data are presented as mean \pm SEM.

from 11 cultures) showed a clear peak in the range of $0.2-0.4 \mathrm{~mm}$ from the pial surface, which corresponds to layer 4 and the lower part of layer 2/3. Moreover, the Monte Carlo analysis demonstrated that this peak was unlikely attributable to the result of random branching along the analyzed parent axons (Fig. $3 B$ ). Thus, thalamic axons formed branches preferentially in the target layer even on fixed cortical tissue.

\section{Biochemical characterization of branching factor}

The above observation indicates that branching is regulated by lamina-specific membrane-associated components because the contribution of diffusible factors that living cortical cells release continuously is excluded in this culture preparation. The biochemical characteristics of the membrane-associated branch-regulating activity were further studied by applying enzymatic treatments to cortical slices before culturing. This manipulation was also expected to reveal whether such molecules act to promote branch addition in layer 4 or to inhibit branching outside layer 4 . In this study chondroitinase, PI-PLC, and endo $\mathrm{N}$ were used because these enzymes cut specific molecular sites in presumptive growth- or branch-regulating factors (Roskies and O'Leary, 1994; Tang et al., 1994; Yin et al., 1995; Emerling and Lander, 1996; Castellani and Bolz, 1998).
The effectiveness of these enzymes at removing their substrate molecules from cortical slices was examined immunohistochemically. As shown in Figure 4, endo $\mathrm{N}$ was found to completely remove PSA immunoreactivity, which is normally associated with NCAMs. Likewise, chondroitinase and PI-PLC treatments were found to reduce chondroitin sulfate and TAG-1 levels, respectively (Yamamoto et al., 2000).

As demonstrated in Figure 5, axonal branches formed in enzymatically treated slices as well as untreated ones, but the number of branches, branch length, and laminar specificity of branch points were different among these groups. These features are further analyzed quantitatively.

First, the average number of branch points per axon was examined (Table 1). A marked change was found after endo $\mathrm{N}$ treatment. The number of branch points in endo $\mathrm{N}$ treatment (22 axons from 11 cultures, three whole and eight cut slices) was increased $>70 \%$ compared with the untreated group (Fig. 5). The number was also slightly increased in chondroitinase (21 axons from eight cultures, two whole and six cut slices) and PI-PLC (26 axons from nine cultures, two whole and seven cut slices) treatments, but the difference was not significant (Table 1). Second, branch tip length was measured in each group. In all treatments branch tip length 




Figure 3. Laminar specificity for pial ingrowth. $A$, Individually distinguishable labeled axons $(n=44)$ were traced from confocal images. Arrows indicate average layer 4 borders of cortical slices $(n=11)$ in the pial arrangement. $B$, Distribution histogram of branching points in the fixed cortical slices. Asterisks indicate significant differences $(p<0.001$ in $0.2-0.3$ $\mathrm{mm}$ from the pia and $p<0.005$ in $0.3-0.4 \mathrm{~mm}$ ) from the value that was predicted by the simulation of random branching.
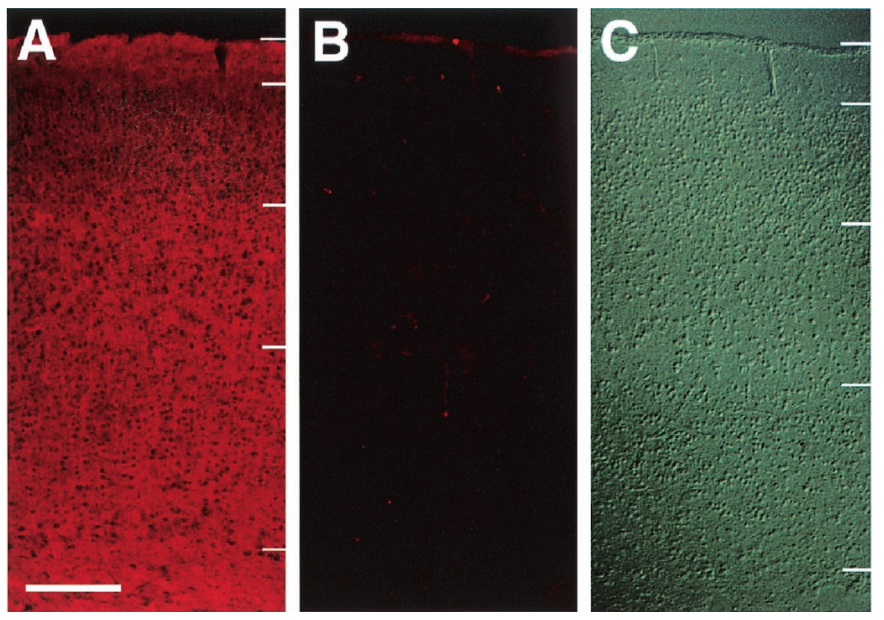

Figure 4. Endo $\mathrm{N}$ treatment of fixed cortical slices. $A$, The distribution of PSA on a cortical section is shown by anti-PSA immunohistochemistry. $\mathrm{Cy} 3$-conjugated secondary antibodies were used for visualization. $B$, After endo $\mathrm{N}$ treatment, PSA immunoreactivity is greatly reduced. $C$, Nomarski image of the same section as shown in $B$. Short bars on the right side indicate laminar boundaries between layers $1,2 / 3-4,5,6$, and the white matter. Scale bar: $A, 200 \mu \mathrm{m}$.

tended to be shorter than in controls, but the decrease was remarkable in chondroitinase and endo $\mathrm{N}$ treatments (Table 1, see also Fig. 5). The average branch tip length was $\sim 50 \%$ shorter in both these treatments than control. On the other hand, total branch length and arbor size did not differ greatly between untreated and treated groups, although the total length was slightly larger in the case of endo $\mathrm{N}$ treatment (Table 1).

Finally, the laminar specificity of branch points was studied with the Monte Carlo analysis, as described above. In all of the treatments, the peaks of the branch distributions were located in layer 4 (Fig. 6). In particular, the laminar distribution of branch points in chondroitinase treatment was quite similar to that in untreated slices. The difference between the value predicted from the simulation and the observed data were highly significant for branches $0.3-0.4 \mathrm{~mm}$ from the pial surface $(p<0.001$; Fig. $6 B$, hatched columns). In this treatment, however, branches tended to form more often in the superficial layers and to be suppressed in the deep layers compared with controls (Fig. 6B, hatched and dotted columns). In PI-PLC treatment, the frequency in and around layer 4 was decreased, whereas branch formation tended to be less inhibited in the deep layers (Fig. 6C, dotted columns), but the experimentally observed branching probability in the target layer was still significantly larger than that expected to occur at random ( $p<0.001$ in the range of $0.3-0.4 \mathrm{~mm}$; Fig. $6 C$, hatched columns). In contrast, the distribution was less concentrated in layer 4 in endo $\mathrm{N}$ treatment (Fig. 6D; see also Fig. 5). In the normal target layer (0.3-0.4 $\mathrm{mm}$ from the pial surface) in endo N-treated cases, the decrease in branch frequency was barely significant by the Monte Carlo analysis $(p<0.05)$, and in all other layers the probability of branching was no longer different from that of the random simulation.

\section{DISCUSSION}

The present study demonstrated that thalamocortical axons in vitro specifically form branches in layer 4 of fixed cortical tissue, suggesting that membrane-bound components such as cell surface or extracellular matrix molecules are responsible for lamina-specific branch formation. In addition, the analysis of branching of thalamic axons on cortical slices subjected to enzymatic treatments that affect the cell surface indicated that a particular component, PSA, may play a role in conferring laminar specificity to thalamocortical axon branch formation by inhibiting axonal branching in inappropriate layers.

\section{Laminar specificity of branch formation on fixed cortex}

A remarkable finding in this study is that, even when grown on fixed cortical slices, thalamic axons largely restricted their branch formation to layer 4. This laminar specificity is quite similar to that found in vivo (Kageyama and Robertson, 1993; Catalano et al., 1996) and in cocultures of thalamic explants with living cortical slices (Yamamoto et al., 1989, 1992, 1997; Molnár and Blakemore, 1991, 1999; Bolz et al., 1992), although other features of the branches are not necessarily the same among these conditions (see below). These findings suggest that at least some of the molecular cues for lamina-specific branch formation are preserved in fixed cortical slices.

Our previous time-lapse study in thalamocortical coculture preparations has demonstrated that the majority of the initial branches of thalamocortical axons emerge in layer 4 (Yamamoto et al., 1997), which suggested the existence of a local signal for laminaspecific branching. The present results further indicate that specific membrane-associated molecules are responsible for this process, because the effects of diffusible factors released from cortical cells are excluded in the present experimental conditions. To date it has been shown that retinal axons preferentially form branches in appropriate layers of fixed tectal tissues (Yamagata and Sanes, 1995) or in the membrane fraction obtained from the corresponding tectal region (Roskies and O'Leary, 1994). Cortical neurons have also been shown to form branches preferentially on membrane obtained from particular layers (Castellani and Bolz, 1997). These findings are consistent with the present results for the thalamocortical projection. 

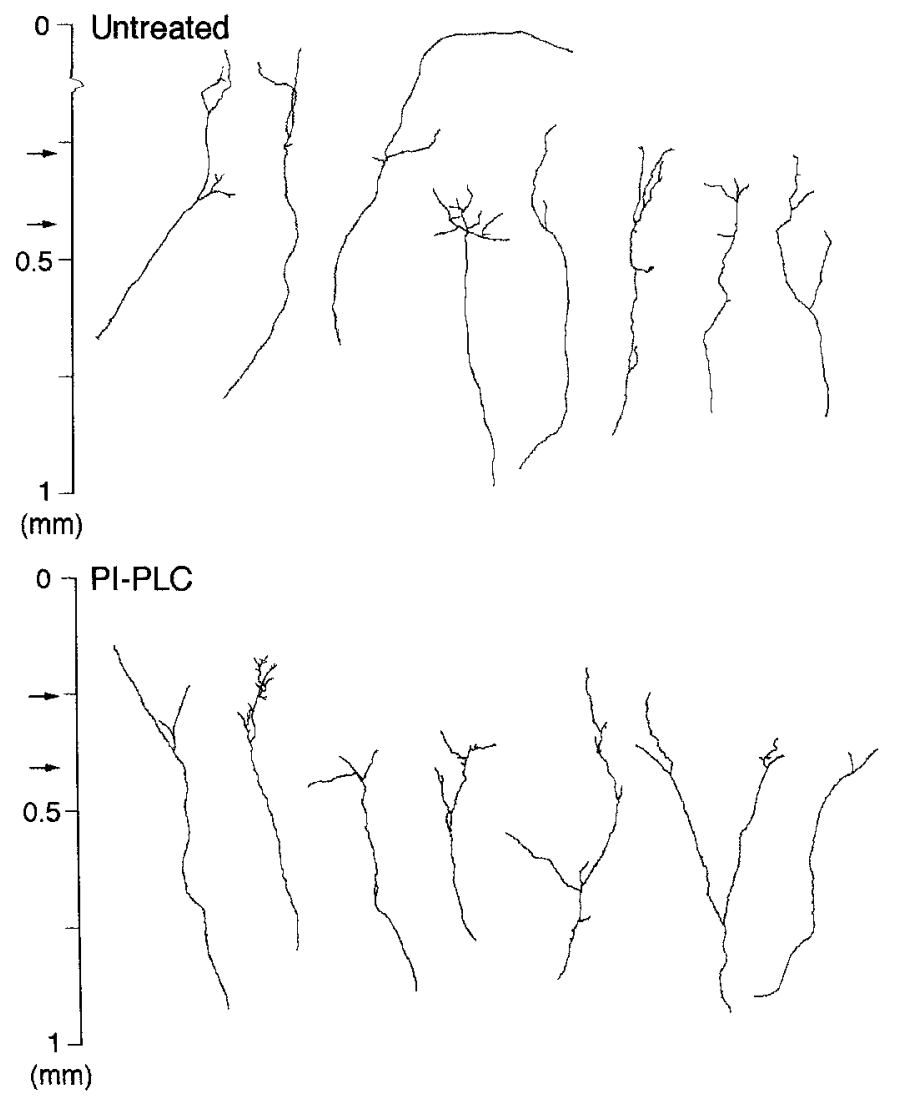
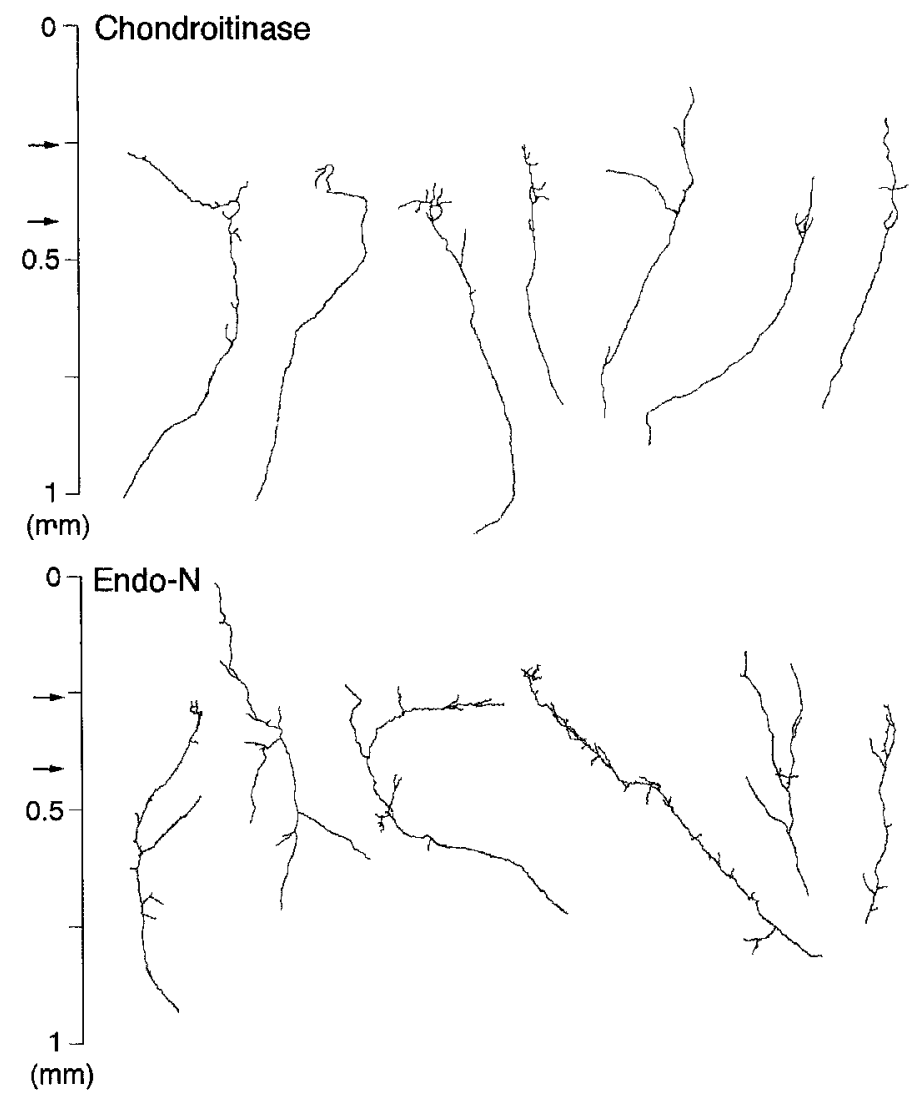

Figure 5. Individual thalamic axons in untreated and enzymatically treated cortical slices. Typical examples are shown in each treatment. Note that the number of branches is increased in endo $\mathrm{N}$ and that the population with short branches appears to be increased by endo $\mathrm{N}$ and chondroitinase treatments.



Figure 6. Laminar distribution of branch points and specificity in enzymatically treated cortical slices. Triple, double, and single asterisks indicate significant differences $(p<0.001, p<0.01$, $p<0.05$, respectively) from the distribution predicted by the simulation of random branching. Hatched and dotted columns represent values that are significantly larger and lower than predicted values, respectively.

\section{Possible mechanisms for lamina-specific branch formation}

In principle, there can be both positive and negative regulation of branch formation. An inhibitory mechanism has been suggested for local cortical circuit formation by the observation that collaterals of developing layer $2 / 3$ neurons emerge only in layers $2 / 3$ and 5 from the outset but never appear in layer 4 (Katz, 1991). Likewise, it has also been shown that layer 6 axons bypass layer 5 and arborize specifically in layer 4 in vivo and in vitro (Callaway and Lieber, 1996; Dantzker and Callaway, 1998). The present study clearly demonstrated the existence of inhibitory regulation of thalamocortical axon branching. Endo $\mathrm{N}$ treatment weakened laminar specificity by increasing branch formation in layers other than layer 4, especially in the deep layers, where the branching frequency in controls was much lower than the value predicted for a random process.

This role for PSA would be easiest to explain whether PSA expression was lowest in layer 4. However, PSA expression is nearly uniform across cortical layers, and therefore a more complex mechanism must be involved. Whereas it is not as yet possible to define this mechanism, one possibility is that a positive cue (see below) whose activity is suppressed by PSA is distributed in all layers but with a peak in layer 4 . If the threshold of activity required for branching is only attained in layer 4 , then in effect PSA serves as a filter that increases the signal-to-noise ratio during innervation.

The logical alternative to inhibition of branch formation would be that PSA promotes elimination of exuberant branches. However, this is unlikely to be the case for thalamocortical axons 
because our previous time-lapse study has shown that both transient and stable branches are located preferentially in layer 4 (Yamamoto et al., 1997). Hence, PSA in the cortex is considered to prevent thalamocortical axons from forming branches in inappropriate layers rather than eliminate side branches that appear in layers other than layer 4.

In accordance with a role for PSA negative regulation of branching, retinal axons defasciculate and sprout inappropriately in their pathway and tectal target regions when PSA is removed by endo $\mathrm{N}$ application in vivo (Yin et al., 1995). Similarly, when PSA is lost from the hippocampus through treatment with endo N, mossy fibers from the dentate gyrus exhibit excessive branch buds in CA3 (Seki and Rutishauser, 1998).

On the contrary, it has been shown that PSA can also enhance defasciculation or branching in motor neuron tracts (Tang et al., 1994). A similar effect was observed in corticopontine projections through removal of PSA (Daston et al., 1996). These actions are opposite to those found in the tectum and hippocampus, as well as our present study of the cortex. An explanation for the opposite influence is that the quantity of PSA in the local environment is originally different between these cases (Yin et al., 1995). Thus, a relatively large amount of PSA in the surrounding cortical cells may suppress thalamocortical axon branching, whereas a low amount would promote defasciculation of motor axons or corticospinal axons. Alternatively, there may be differences in the expression of molecules that are influenced by PSA.

In addition to the inhibitory mechanism, it is possible that a positive regulator is expressed in the target layer (Del Rio et al., 1997), because lamina-specific branch formation of thalamocortical axons was not abolished completely by endo $\mathrm{N}$ application. Although we could not find any enzymatic treatment that decreased the number of branch points in layer 4 , the fact that most thalamic axons that grew outside the cortical slice extended long distances with little branching (data not shown) indicates that there probably exist some membrane-associated factors in the cortex that can promote branch formation. Castellani and Bolz (1998) have shown that axonal branches from layer 6 neurons are increased when grown on the membrane fraction of their natural target layer 4 or on membranes from ephrin-A5-expressing cells, suggesting that an EphA system induces axonal branches in a subset of cortical neurons. This supports the presence of a positive regulator of branching, at least for cortical axons, although PI-PLC treatment did not change branch formation dramatically in the present study.

\section{Branch growth}

Quantitative analysis demonstrated that branch tip length was decreased substantially by endo $\mathrm{N}$ and chondroitinase treatments, although the number of branch points and the laminar specificity were not changed in chondroitinase treatment. This result suggests that PSA and chondroitin sulfate may affect branch growth by controlling cellular adhesion (Emerling and Lander, 1996). In particular, the fact that axonal growth was decreased slightly in the deep layers of neuramindase-treated fixed cortical slices in the previous study (Yamamoto et al., 2000) suggests that PSA provides a growth-permissive environment both for branches as well as for the primary axonal shaft, whereas this moiety operates as a negative regulator for inappropriate branch emergence.

Another observation regarding branch growth was that the arbor sizes and the total axonal lengths did not differ significantly among untreated and treated groups (Table 1), suggesting that the overall growth of thalamic axons in the cortex may not be influenced greatly by membrane-bound molecules. Under living conditions, growth factors such as neurotrophins may be released from cortical cells and promote growth and complexity of branches (Rennie et al., 1994; Cohen-Cory and Fraser, 1995; Sawai et al., 1996; Castellani and Bolz, 1999). In accordance with this view, the branch tip lengths $(45-90 \mu \mathrm{m})$ and the arbor sizes $(140-160 \mu \mathrm{m})$ in fixed cortex (Table 1), regardless of treatments, are substantially smaller than those in the living cultured cortex $(227 \pm 28$ and $276 \pm 52 \mu \mathrm{m}$, respectively, calculated from the data of Yamamoto et al., 1997), although the number of branch points is in the same range $(3.7 \pm$ 0.5 vs $2.9 \pm 0.7)$. Arbor sizes in the fixed cortex are also lower than those reported in P7 somatosensory cortex in vivo $(228.5 \pm 23 \mu \mathrm{m}$; Catalano et al., 1996).

\section{Branch formation and axonal termination in the target layer}

It is worthwhile to note the relation between termination and branch formation of thalamocortical axons in the target layer. Our recent study, which also used fixed cortical slices, suggested that GPI-anchored growth-inhibitory molecules are expressed in the upper layers and contribute to lamina-specific axonal termination (Yamamoto et al., 2000). On the contrary, the same enzymatic treatment did not produce an obvious change of lamina-specific branch formation. This suggests that target-dependent branching is regulated separately from axonal termination, which is consistent with our observations of the behaviors of thalamocortical axons growing in living cortex in a previous time-lapse study (Yamamoto et al., 1997). However, it is also possible that these two axonal behaviors take place in association with each other, even though they are regulated separately (Yamamoto et al., 1997; Szebenyi et al., 1998). In the retinotectal projection, positional cues for topographic projections appear to coexist or highly correlate with those inducing collateral formation (Roskies and O'Leary, 1994).

It should be noted that the present method may not reveal all molecular features of branching and growth. The signaling of different molecules is likely to be differentially susceptible to fixation. Moreover, molecular identification by enzymatic treatment, except for endo $\mathrm{N}$, is ambiguous, because a large class of molecules is degraded by any single enzyme. Conversely, there is a possibility that a very small amount of residual substrate below the threshold for immunohistochemical detection, might still have some effects on axonal behaviors. Another potential problem is a sampling bias in collecting thalamic axons (see Materials and Methods), which may dilute some effects on axonal growth. Indeed, the increase in axonal growth after PI-PLC treatment (Yamamoto et al., 2000) was not observed in individual axons, which is considered to be primarily attributable to the sampling bias, because the gross growth appeared to be higher in PI-PLC-treated than untreated slices.

Despite the above problems, the present study demonstrates that membrane-associated molecules contribute to lamina-specific branch formation of thalamocortical axons and that PSA acts as an inhibitory regulator. Further attempts, such as searching for layerspecific molecules, will be necessary to reveal the complete molecular mechanisms underlying laminar specificity.

\section{REFERENCES}

Agmon A, Yang LT, O’Dowd DK, Jones EG (1993) Organized growth of thalamocortical axons from the deep tier of terminations into layer IV of developing mouse barrel cortex. J Neurosci 13:5365-5382.

Bolz J, Novak N, Staiger V (1992) Formation of specific afferent connections in organotypic slice cultures from rat visual cortex with lateral geniculate nucleus. J Neurosci 12:3054-3070.

Callaway EM, Lieber JL (1996) Development of axon arbors of layer 6 pyramidal neurons in ferret primary visual cortex. J Comp Neurol 376:295-305.

Castellani V, Bolz J (1997) Membrane-associated molecules regulate the formation of layer-specific cortical circuits. Proc Natl Acad Sci USA 94:7030-7035

Castellani V, Bolz J (1999) Opposing roles for neurotrophin-3 in targeting and collateral formation of distinct sets of developing cortical neurons. Development 126:3335-33345.

Castellani V, Yue Y, Gao P-P, Zhou R, Bolz J (1998) Dual action of a ligand for tyrosine kinases on specific populations of axon during the development of cortical circuits. J Neurosci 18:4663-4672.

Catalano SM, Robertson RT, Killackey HP (1996) Individual axon morphology and thalamocortical topography in developing rat somatosensory cortex. J Comp Neurol 366:36-53.

Cohen-Cory S, Fraser S (1995) Effects of brain-derived neurotrophic factor on optic axon branching and remodelling in vivo. Nature 378:192-196.

Dantzker JL, Callaway EM (1998) The development of local, layerspecific visual cortical axons in the absence of extrinsic influences and intrinsic activity. J Neurosci 18:4145-4154.

Daston MM, Bastmeyer M, Rutishauser U, O'Leary DD (1996) Spatially restricted increase in polysialic acid enhances corticospinal axon branch- 
ing related to target recognition and innervation. $\mathrm{J}$ Neurosci 16:5488-5497.

Del Rio JA, Heimrich B, Borrell V, Forster E, Drakew A, Alcantara S, Nakajima K, Miyata T, Ogawa M, Mikoshiba K, Derer P, Frotscher M, Soriano E (1997) A role for Cajal-Retzius cells and reelin in the development of hippocampal connections. Nature 385:70-74.

Donoghue MJ, Rakic P (1999) Molecular evidence for the early specification of presumptive functional domains in the embryonic primate cerebral cortex. J Neurosci 19:5967-5979.

Emerling DE, Lander AD (1996) Inhibitors and promoters of thalamic neuron adhesion and outgrowth in embryonic neocortex: functional association with chondroitin sulfate. Neuron 17:1089-1100.

Gao PP, Yue Y, Zhang JH, Cerretti DP, Levitt P, Zhou R (1998) Regulation of thalamic neurite outgrowth by the Eph ligand ephrin-A5: implications in the development of thalamocortical projections. Proc Natl Acad Sci USA 95:5329-5334.

Ghosh A, Shatz CJ (1992) Pathfinding and target selection by developing geniculocortical axons. J Neurosci 12:39-55.

Gilbert CD (1983) Microcircuitry of the visual cortex. Annu Rev Neurosci $6: 217-247$.

Godement P, Vanselow J, Thanos S, Bonhoeffer F (1987) A study in developing visual systems with a new method of staining neurones and their processes in fixed tissue. Development 101:697-713.

Jones EG (1981) Anatomy of cerebral cortex: Columnar input-output organization. In: The organization of the cerebral cortex (Schmitt FO, Worden FG, Adelman G, Dennis SG, eds), pp 199-235. Cambridge, MA: MIT.

Kageyama GH, Robertson RT (1993) Development of geniculocortical projections to visual cortex in rat. J Comp Neurol 335:123-148.

Katz LC (1991) Specificity in the development of vertical connections in cat striate cortex. Eur J Neurosci 3:1-9.

Lund RD, Mustari MJ (1977) Development of geniculocortical pathway in rats. J Comp Neurol 173:289-306.

Mackarehtschian K, Lau CK, Caras I, McConnell SK (1999) Regional differences in the developing cerebral cortex revealed by ephrin-A5 expression. Cereb Cortex 9:601-610.

Molnár Z, Blakemore C (1991) Lack of regional specificity for connections formed between thalamus and cortex in coculture. Nature 351:475-477.

Molnár Z, Blakemore C (1999) Development of signals influencing the growth and termination of thalamocortical axons in organotypic culture. Exp Neurol 156:363-393.

Rennie S, Lotto RB, Price DJ (1994) Growth-promoting interactions between the murine neocortex and thalamus in organotypic co-cultures. Neuroscience 61:547-564.
Roskies AL, O'Leary DDM (1994) Control of topographic retinal axon branching by inhibitory membrane-bound molecules. Science 265:799-803.

Sawai H, Clarke DB, Kittlerova P, Bray GM, Aguayo AJ (1996) Brainderived neurotrophic factor and neurotrophin- $4 / 5$ stimulate growth of axonal branches from regenerating retinal ganglion cells. J Neurosci 16:3887-3894.

Seki T, Arai Y (1991) Expression of highly polysialylated NCAM in the neocortex and piriform cortex of the developing and the adult rat. Anat Embryol (Berl) 184:395-401.

Seki T, Rutishauser U (1998) Removal of polysialic acid-neural cell adhesion molecule induces aberrant mossy fiber innervation and ectopic synaptogenesis in the hippocampus. J Neurosci 18:3757-3766.

Szebenyi G, Callaway JL, Dent EW, Kalil K (1998) Interstitial branches develop from active regions of the axon demarcated by the primary growth cone during pausing behaviors. J Neurosci 18:7930-7940.

Tang J, Rutishauser U, Landmesser L (1994) Polysialic acid regulates growth cone behavior during sorting of motor axons in the plexus region. Neuron 13:405-414.

Wang KH, Brose K, Arnott D, Kidd T, Goodman CS, Henzel W, TessierLavigne M (1999) Biochemical purification of a mammalian slit protein as a positive regulator of sensory axon elongation and branching. Cell 19:771-784.

Yamagata M, Sanes JR (1995) Lamina-specific cues guide outgrowth and arborization of retinal axons in the optic tectum. Development 121:189-200.

Yamamoto N, Kurotani T, Toyama K (1989) Neural connections between the lateral geniculate nucleus and visual cortex in vitro. Science 245:192-194.

Yamamoto N, Yamada K, Kurotani T, Toyama K (1992) Laminar specificity of extrinsic cortical connections studied in coculture preparations. Neuron 9:217-228.

Yamamoto N, Higashi S, Toyama K (1997) Stop and Branch behaviors of geniculocortical axons: a time-lapse study in organotypic cocultures. J Neurosci 17:3653-3663.

Yamamoto N, Inui K, Harada A, Murakami F, Hanamura K (1998) Regulation of lamina-specific branching of thalamocortical axons. Soc Neurosci Abstr 24:288.

Yamamoto N, Matsuyama Y, Harada A, Inui K, Murakami F, Hanamura K (2000) Characterization of factors regulating lamina-specific growth of thalamocortical axon. J Neurobiol 42:56-68.

Yin X, Watanabe M, Rutishauser U (1995) Effect of polysialic acid on the behavior of retinal ganglion cell axons during growth into the optic tract and tectum. Development 121:3439-3446. 